\title{
PISCINE UDP-GLUCURONOSYLTRANSFERASE 1B
}

\author{
Michael J. Leaver, Joy Wright, Evridiki Boukouvala* and Stephen G. George ${ }^{1}$ \\ Institute of Aquaculture, University of Stirling, Stirling FK9 4LA, UK \\ * Fisheries Research Institute, National Agricultural Research Foundation, Nea Peramos, \\ 64007 Kavala, Greece
}

Email: sg21@stir.ac.uk Tel: $01786467927 \quad$ Fax: 01786472133

\begin{abstract}
Glucuronidation is an important detoxification pathway for organic pollutants in fish. We report here the isolation and characterisation of UDP-glucuronosyltransferases (UGT) genes from the closely related marine flatfish, plaice (Pleuronectes platessa) and flounder (Platichthys flesus). The deduced amino acid sequences share greater similarity with mammalian UGT1 family genes than UGT2 genes (44-47\% and 39-40\% amino acid identity respectively) and have been designated UGT1B. Both plaice and flounder UGT1B mRNAs are most highly expressed in liver, but are also expressed in intestine, gill, kidney and adipose tissue to a greater extent than muscle, heart or brain. Plaice UGT1B mRNA was undetectable in gametes or fertilised eggs and there was a large increase in expression between gastrulation and myotome formation after which levels declined some 5-10 fold. Flounder UGT1B mRNA was increased in liver after intraperitoneal injection of Arochlor 1254 or lindane, but not after perflourooctanoic acid or 3-methylcholanthrene. In isolated flounder hepatocytes UGT1B mRNA was increased by benzo(a)pyrene but not by ethynylestradiol. Expression of a cDNA for plaice UGT1B in cos7 cells resulted in higher 1-naphthol conjugation in cell homogenates compared to steroid conjugation, whilst bilirubin and bile acid conjugation undetectable. This indicates that the plaice gene codes for the phenol-conjugating UGT previously purified in our laboratory from this species and that it is likely to play a major role in the detoxification of polyaromatic hydrocarbons in flatfish. Its role in development is unknown.

UGT1B genes are also present in pufferfish (Tetraodon nigroviridis) and zebrafish (Danio rerio) genomes, but that they differ in their genic organisation. Pufferfish possess multiple (repeated) complete UGT1 genes and Southern blots indicate that the homologous plaice UGT1B gene may also be organised in this way. In contrast, zebrafish appear to have two UGT1 loci whose sequences and intron/exon structures are closely related to that of plaice, however, the organisation of these genes is similar to the mammalian UGT1 family since each has multiple repeated exon 1's which are alternatively spliced to a common set of exons encoding the aglycone binding domain. Taken together with evidence from phylogenetic comparison of fish sequences with UGT1 and UGT2 families in mammals, we suggest these homologous fish UGTs should all be included within the vertebrate UGT1 family and designated as UGT1B.
\end{abstract}

\section{KEY WORDS}

UDP-glucuronosyltransferase, detoxification, fish, gene, UGT

\section{FOOTNOTE}

Abbreviations used: UGT, UDP-glucuronosyltransferase; UDP, uridine diphosphate; CYP1A, cytochrome P4501A; CMP, cytidine monophosphate: ER, endoplasmic reticulum; EST, expressed sequence tag; PCR, polymerase chain reaction; PBS, phosphate buffered saline; 
FBS, fetal bovine serum; DMEM, Dulbeccos modified Eagles medium; HBSS, Hanks balanced salt solution.

1 To whom correspondence should be addressed (e-mail: sgg1@stir.ac.uk.

\section{INTRODUCTION}

Glucuronide formation is a major mammalian pathway in the biotransformation and elimination of a broad range of potentially toxic endogenous and xenobiotic organic compounds. Glucuronidation reactions are catalysed by a superfamily of endoplasmic reticulum-bound enzymes, the UDP-glucuronosyltransferases (EC 2.4.1.17), which conjugate UDP-glucuronic acid with lipophilic acceptor substrates which include steroids, bile acids, bilirubin and a diverse array of drugs and environmental contaminants (Dutton, 1980). UDPglucuronosyltransferases belong to a superfamiliy of glycosyltransferases termed UGTs and in mammals glucuronide conjugation is carried by members of the UGT1 and UGT2 subfamilies (Mackenzie et al, 2005). UGT1 and UGT2 enzymes have attracted attention because of their importance in drug metabolism (Mulder, 1992) and the discovery that a variety of hereditary diseases of humans are caused by defects in UGT activity (Owens et al., 1992). Moreover, polymorphisms of these genes can give rise to significant pharmacological impacts in humans with respect to cancer risk and drug-induced toxicity (Guillemette, 2003). Purification and catalytic reconstitution of these membrane bound enzymes is difficult (Radominska-Pandya, et al., 2005) and therefore, much of the progress in elucidating the function of mammalian UGT isoforms has been achieved through gene cloning and heterologous expression studies (Radominska-Pandya et al., 2005). UGT1 proteins are primarily involved in the conjugation of bilirubins, phenols, amines and carboxylic acids whilst UGT2 proteins appear to be particularly involved in steroid and bile acid conjugation (Tukey and Strassburg, 2000). Although each member of the family of mammalian UGTs glucuronidates a distinct set of substrates, there is a great deal of overlap with some forms exhibiting a relatively narrow range of specificities and others having the capability to conjugate several different compounds. In addition, the levels of some mammalian UGT isoforms can be modulated by administration of a variety of hormones and xenobiotics. The modulation of UGT levels by hormones, as well as defects in glucuronidating capacity are now known to be dependent on the structures, sequences and regulatory regions of UGT1 and UGT2 genes (Chen et al., 2005; Mackenzie et al., 2003)

Interestingly, glucuronidation reactions appear to be restricted to vertebrates and have not yet been identified in invertebrates or plants where glucosylation is utilised for analogous detoxification reactions (Dutton, 1980) From an evolutionary point of view, it is therefore of interest to characterise UGTs from lower vertebrates. To date the most 'primitive' organisms which have been established as having glucuronidating capacity are fish species (Clarke et al, 1991). Studies of glucuronidation in fish have been predominantly directed toward understanding more about the potential of fish to deal with organic pollutants since the coasts and estuaries of many major industrialised areas are subject to increasing levels of environmental contamination by a variety of chemical waste products. Both plaice and flounder are utilized in Western European statutary biomonitoring programs as bioindicator species. Previous studies on the xenobiotic metabolising enzyme systems of plaice have revealed that glucuronidation is an important detoxification pathway, particularly for lipophilic aromatic phenolic compounds. Such compounds are common carcinogenic contaminants in themselves and also arise as a consequence of the oxidative metabolism of polyaromatic hydrocarbons (Clarke et al., 1991; George, 1994; Leaver et al., 1992). 
Studies on piscine UGTs, as with earlier mammalian studies, have been forestalled by the difficulty in dealing with tightly membrane bound, multiple UGT isozymes and thus far only one enzyme, a phenol-conjugating isoform, has been purified to homogeneity (Clarke et al., 1992). We have previously isolated a partial length plaice UGT cDNA (Pirrit et al., 1995) originally designated as a member of the UGT1 family, UGT1B1 (Mackenzie et al., 1998). In addition, the organization of the plaice UGT gene and evidence of considerable allelic variation has been reported (George et al, 1998; George and Leaver, 2002). In order to extend these studies we have undertaken further cDNA and gene cloning, tissue expression, catalytic characterization and phylogenetic comparisons of this gene and enzyme from plaice and from the closely related flounder.

\section{METHODS}

\section{Animals and Experimental Treatments}

Juvenile plaice and flounder were obtained by trawl from the Scottish East coast and kept in filtered recirculating seawater at $10^{\circ} \mathrm{C}$ for two weeks before use. Animals were fed ad libitum during this period. Gonadally immature male flounder were injected IP with $100 \mathrm{mgKg}^{-1}$ perfluorooctanoic acid, $50 \mathrm{mgKg}^{-1}$ Arochlor $1254,5 \mathrm{mgKg}^{-1}$ lindane, $25 \mathrm{mgKg}^{-1} 3$ methylcholanthrene or $1 \mathrm{mLKg}^{-1}$ olive oil (vehicle control). Animals were sacrificed 48 hours after treatment and livers taken for RNA preparation. Pooled eggs from 3 female plaice were fertilized with pooled milt from 3 males and reared to metamorphosis at $8^{\circ} \mathrm{C}$ in a flowing sea water aquarium system. Upon yolk resorbtion larvae were fed brine shrimp (Artemia salina). Embryos and larvae were sampled intervals up to metamorphosis. This experiment was performed twice in subsequent years.

\section{Hepatocyte Preparation and Treatment}

Livers from six juvenile male flounder (15-25g) were removed under aseptic conditions, rinsed in 3 volumes of PBS, $100 \mathrm{UmL}^{-1}$ Penicillin, $100 \mu \mathrm{gmL}^{-1}$ Streptomycin, $200 \mathrm{UmL}^{-1}$ Kanamycin (PBS/PSK) at $10^{\circ} \mathrm{C}$. The livers were then finely chopped to approximately $2 \mathrm{~mm}$ dice and incubated, shaking gently, for $15 \mathrm{~min}$ in PBS/PSK at $10^{\circ} \mathrm{C}$. Liver fragments were then allowed to settle and the medium decanted and replaced with HBSS/PSK, $2 \mathrm{mM} \mathrm{CaCl} 2$. This suspension was then incubated, shaking gently, for $15 \mathrm{mins}$, before replacing the medium with HBSS/PSK/Ca containing $0.5 \%$ collagenase (Sigma, Poole, UK) and incubating at $10^{\circ} \mathrm{C}$ for 3 hours. The resulting cell suspension was then filtered sequentially through sterile nylon gauze of $0.5 \mathrm{~mm}$ and $0.1 \mathrm{~mm}$ gauge. The filtrate was spun for $5 \mathrm{mins}$ at $15 \mathrm{~g}$ to pellet the majority of the erythrocytes, followed by centrifugation of the supernatant for $5 \mathrm{~min}$ at $300 \mathrm{~g}$ to pellet hepatocytes. The hepatocyte pellet was resuspended in DMEM/PSK, 10\% fetal bovine serum. After counting and adjusting volume, cells were distributed to six-well plastic tissue culture plates ( $2 \mathrm{~mL}$ per well, $10^{6}$ cells per well). Plates were incubated at $15^{\circ} \mathrm{C}$ in $4 \% \mathrm{CO}_{2}$ for 16 hours before adding, to three replicate wells each, $5 \mu \mathrm{L}$ of DMSO (vehicle control), $10 \mu \mathrm{M}$ ethynylestradiol or $50 \mu \mathrm{M}$ benzo(a)pyrene. After 24 hours cells and media were removed by gentle scraping and transferred to $2 \mathrm{ml}$ microcentrifuge tubes. Cell suspensions were then pelleted at $100 \mathrm{~g}$ for 3 mins, gently resuspended in $2 \mathrm{~mL}$ of PBS and pelleted again. All traces of PBS were then removed by aspiration and the cell pellets resuspended and lysed by vigorous vortex mixing in $200 \mu \mathrm{L}$ of a guanidinium salt/phenol-based RNA extraction reagent (TriReagent, Sigma, Poole, UK). Hepatocyte viability was assessed in duplicate in culture plates by Trypan blue exclusion immediately after plating and after 40 hours. Over this period viability dropped from an average of $78 \%$ to $64 \%$ with no measurable differences between treatments. 


\section{Nucleic acid preparation and hybridisation.}

DNA isolation, RNA isolation, blotting and hybridisation procedures have been described previously (Leaver et al., 1997). Isolation of the plaice UGT gene has been described previously (George et al., 1998; EMBL/Genbank accession no. AJ249082) and the flounder gene (EMBL/Genbank submitted) was isolated using an identical method, by screening a flounder genomic DNA library with the full length plaice UGT cDNA. The synthesis and sequence of plaice UGT cDNA has been described previously (George and Leaver, 2002; EMBL/Genbank accession no. AJ249081). Northern and Southern blots were hybridized to a ${ }^{32}$ P-labeled DNA fragment corresponding to the aglycone-binding domain encoded by exon1 of the plaice UGT gene (George et al., 1998, Fig. 1). Northern blots of embryo-larval samples were quantified by densitometic scanning of autoradiographs (Shimadzu Model CS9000 dual wavelength flying spot densitometer) and normalised to the ethidium bromide-stained $28 \mathrm{~S}$ RNA band visualised under UV illumination since tubulin mRNA levels were very low compared with 28S RNA levels at fertilisation and rose to a constant ratio by hatching.

\section{Sequence Analysis and Phylogenetic Comparisons}

Plaice UGT1B cDNA and flounder genomic DNA was ligated to pBluescript KS+ or pcDNA3 and amplified in E. coli. Purified plasmids were sequenced using oligonucleotide primers, BigDye sequencing reagents and an ABI 377 autosequencer (Applied Biosystems inc.), and sequences were compiled and verified using ABI Autoassembler software. The sequence of the plaice UGT cDNA was used to search the ENSEMBL (www.ensembl.org) genome databases of the zebrafish (Danio rerio, assembly Zv5, www.ensembl.org) and pufferfish (Tetraodon nigrivridis, assembly TETRAODON 7, www.ensemble.org) using TBLASTX. Contiguous segments of genomic sequence which showed high levels of homology were further analysed by locating open reading frames and predicting exons and coding amino acid sequences by comparison with plaice UGT. Those exons comprising regions with homology to the first exon (the aglycone-binding domain coding sequence) of plaice UGT1B were used to search EMBL/Genbank databases using BLASTN. These sequences were also translated to amino acid sequence and compared using ClustalX (www.ebi.ac.uk) to generate a multiple alignment. A phylogenetic tree was generated in Clustal using the neighbour joining method of Satou and Nei, (1987). The tree was bootstrapped through 1000 iterations and plotted using Njplot (www.ebi.ac.uk). The sequence of the human UGT2B4 aglycone binding domain was included in the comparison to provide a root for the phylogenetic tree.

\section{Quantitative PCR}

cDNA was synthesized from total plaice or flounder RNA using a commercially available kit according the manufacturers instructions (Reverse-iT Max $1^{\text {st }}$ strand synthesis kit, ABgene, Epsom, UK) from $1 \mu \mathrm{g}$ of total RNA in a reaction volume of $20 \mu \mathrm{L}$ containing $300 \mathrm{ng}$ of random hexamers and 125ng of anchored oligo-dT. Following cDNA synthesis at $42^{\circ} \mathrm{C}$ for 1 hour reactions were stopped by heating at $75^{\circ} \mathrm{C}$ for $10 \mathrm{~min}$ and cDNA diluted to $200 \mu \mathrm{L}$ total volume with water. Quantitative PCR was performed on $2 \mu \mathrm{L}$ of each diluted cDNA sample (equivalent to 10ng of input RNA) and reaction volumes of $20 \mu \mathrm{L}$ contained $10 \mu \mathrm{L}$ of commercially available Taq Polymerase/SYBR Green mastermix (Absolute QPCR SYBR Green Mix, ABgene,Epsom, UK), and 70nM each of gene-specific primers (see below). PCR 
was undertaken in 96 well plates in a Techne Quantica thermocycler. Reactions were initiated by heating to $95^{\circ} \mathrm{C}$ for 15 min followed by 45 cycles of $95^{\circ} \mathrm{C}$ for $15 \mathrm{sec}$ and $60^{\circ} \mathrm{C}$ for $1 \mathrm{~min}$. After 45 cycles a melt curve was generated by measuring sample fluorescence during heating from $75^{\circ} \mathrm{C}$ to $95^{\circ} \mathrm{C}$. Following PCR, five samples derived from each set of primers were chosen at random, and analysed by agarose gel electrophoresis and DNA sequencing. There were no indications of anything other than the predicted products following quantitative PCR, as determined by single peaks in melt curves, single fragments of predicted size in gel electrophoresis and predicted sequences of products (not shown). For comparison of UGT tissue expression levels UGT mRNA was quantified by comparison to a standard sample set of serially diluted, linearized plasmids containing UGT cDNA and then normalized to $18 \mathrm{~S}$ RNA copy number, also quantified by comparison to a standard sample set of serially diluted, linearized plasmids containing a flounder 18S RNA sequence (Genbank/EMBL accession no. DV566337; primers, CTGCCCTATCAACTTTCGATGGTACT and AAAGTGTACTCATTCCAATTACAGGG). Relative expression of UGT (EMBL/Genbank ????; $\quad$ primers AGTCATGGGGCTCTCTGGCTCT GTTACCCTTTGAGGAATTTGCCG) and CYP1A (EMBL/Genbank accession no. AJ132353; primers CAACCATGATCCAGAGCTGTG and GATTATTCTTCCTCCACTGACTCT) mRNA levels between different IP or hepatocyte treatments were calculated using the "delta-delta Ct" method (Pfaffl, 2001) where $\beta$-actin (EMBL/Genbank accession no. AF171095; primers GACCACTGGGATGACATGG and GCGTACAGGGACAGCACAGC)was used as reference gene. Amplification efficiencies for all primer pairs were between 1.96 and 2.08.

\section{Transient expression of plaice UGT1B}

Transient expression of plaice UGT1B cDNA in cos7 cells was carried out by transfection with pcDNA3UGT1B. pcDNA3UGT1B was constructed by excising the entire intact plaice UGT RT-PCR product from pBluescript $\mathrm{KS}+$ and then ligating to the corresponding restriction sites of pcDNA3 (Pharmacia). The integrity of the resulting construct was verified

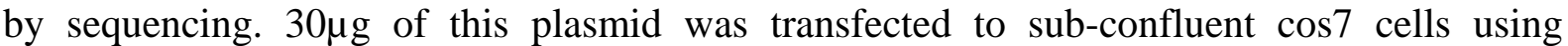
Superfect (Qiagen) according to the suppliers instructions. 30 $\mu$ g of pcDNA3 was used to undertake a parallel series of control transfections. Cells were maintained in L15 (Sigma) medium in closed $175 \mathrm{~cm} 2$ tissue culture flasks. Cells were harvested after 50 hours by scraping, and after washing in PBS, were disrupted by rotating probe homogeniser (Ultraturrax T8) in PBS and the resulting homogenate used immediately for assay.

\section{Catalytic Assays}

Glucuronidating activity of the recombinant protein was measured as described by Matern et al. (1994) using either $0.5 \mathrm{mM}$ 1-naphthol, $0.25 \mathrm{mM}$ bilirubin, $0.1 \mathrm{mM}$ testosterone, $0.1 \mathrm{mM}$ estradiol or $0.5 \mathrm{mM}$ bile acids as acceptor substrates and $1 \mathrm{mM}{ }^{14} \mathrm{C}$-UDP-glucuronate (ARC) as donor substrate. Incubations were carried out in a volume of $50 \mu \mathrm{l}$ containing, in addition to acceptor substrate, $10 \mu \mathrm{l}$ of sample $(10 \mu \mathrm{l}$ of cell homogenate) $100 \mathrm{mM}$ Tris, $\mathrm{pH} 7.4,5 \mathrm{mM}$ $\mathrm{MgCl}_{2}, 1 \mathrm{mM} \mathrm{CMP}, 1 \mathrm{mM}{ }^{14} \mathrm{C}-\mathrm{UDP}$-glucuronic acid $(2 \mu \mathrm{Ci} / \mu \mathrm{mol})$. Incubations were carried out for $30 \mathrm{~min}$ at $30^{\circ} \mathrm{C}$. Assays were performed in quadruplicate for each acceptor substrate and corresponding blank. Blanks contained no acceptor substrate. Reactions were stopped by the addition of $50 \mu \mathrm{l}$ of $0.7 \mathrm{M}$ glycine $/ \mathrm{HCl}, \mathrm{pH} 2,1 \%(\mathrm{v} / \mathrm{v})$ Triton $\mathrm{X}-100$. Glucuronide conjugates were extracted by the addition and vortex mixing ( $1 \mathrm{~min})$ of $500 \mu \mathrm{l}$ of ethyl acetate, $250 \mu \mathrm{l}$ of which was analysed by liquid scintillation spectroscopy. Results were normalised to homogenate protein concentration. The inclusion of Lubrol PX up to $0.4 \mathrm{mg} / \mathrm{mg}$ 
Running title: Piscine UDP-glucuronosyltransferase gene

protein in assays had no effect on activities. Above $0.4 \mathrm{mg}$ Lubrol PX/mg protein activity was inhibited (not shown).

\section{RESULTS}

\section{Sequence and Phylogenetic Analysis of Piscine UGT1B Genes}

We have isolated a 1655nt cDNA for the plaice UGT1B and a homologous gene from flounder both of whose deduced amino acid sequences (530 amino acids with a predicted molecular weight of $60066 \mathrm{Da}$ ) are similar to all other known UGT proteins. The deduced amino acid sequence of the plaice UGT1B cDNA and the flounder cDNA predicted from the gene sequence share $84 \%$ identity. In addition these flatfish proteins share extensive sequence similarities to various mammalian UGTs and other putative piscine UGTs (Fig.1). UGT proteins can be conceptually divided into two domains with the amino-terminal half of the protein, determining aglycone specificity, demonstrating greater sequence divergence between isoforms. The carboxyl-terminal half, believed to contain the binding site for UDPglucuronic acid, displays a higher degree of conservation between different UGT isoforms. UGT is localized to the endoplasmic reticulum (ER) and spans the membrane with a type I topology. The putative transmembrane domain is located near the carboxyl terminus of the protein such that only a small portion of the protein resides in the cytosol. This cytosolic tail is believed to contain an ER-targeting signal which is a putative ER membrane targeting signal. The major portion of the protein is located in the ER lumen, including the proposed substrate-binding domains and the catalytic site (Meech et al, 1997; Radominska-Pandya et al., 2005). These features are clearly displayed by the flatfish UGT1B polypeptides which display between 50 and 55\% identity between the C-terminal half of the flatfish UGT1B polypeptides and the equivalent region of mammalian UGT isoforms. Moreover the flatfish polypeptides contain a potential dilysine ER membrane tag motif, KTKSE (Fig. 1) which is close to a hydrophobic amino acid-rich region. Hydrophobicity plots (not shown), also display a very similar secondary structure to mammalian UGT sequences. The $\mathrm{N}$ - terminal region of the flatfish proteins are more divergent when compared with the equivalent regions of mammalian UGT polypeptides, sharing only 33-40\% identity and 30-33\% identity with UGT1A and UGT2 sequences respectively, however, they contain motifs shared by all other UGTs in their N-terminal domains, including a signal peptide (Fig 1, (Senay et al., 1997; Radominska-Pandya et al., 2005).

Much higher identity in this aglycone-binding region is evident when comparisons are made between the plaice, flounder, zebrafish and pufferfish sequences. Phylogenetic comparisons of the complete deduced amino acid sequence of plaice UGT1B with various mammalian UGT sequences confirmed that plaice UGT1B is more closely related to mammalian UGT1 (44-47\% total identity) than UGT 2 family genes (39-40\% total identity) and this similarly is also borne out by the common 5 exon structure of this plaice gene (George et al., 1998; George and Leaver, 2002) and mammalian UGT1 family genes compared with the 6-exon structure of mammalian 2 family genes. Hence its designation as UGT1B (Mackenzie et al., 1998). Searches of the available genomic sequence for Tetraodon nigriviridis (pufferfish) and Danio rerio (zebrafish) using the plaice UGT1B sequence revealed the presence of multiple closely related genes with higher similarity to the plaice UGT1B than any mammalian UGTs in both species. In the pufferfish (both T. nigroviridis and Takafugu rubripes) genomes, genes related to plaice UGT1B were present as discrete units on two different loci. The first, on chromosome 2, comprised at least two, and probably four, complete repeated UGT genes with the identical 5-exon structure to plaice UGT1B. The second T. nigroviridis locus, on 


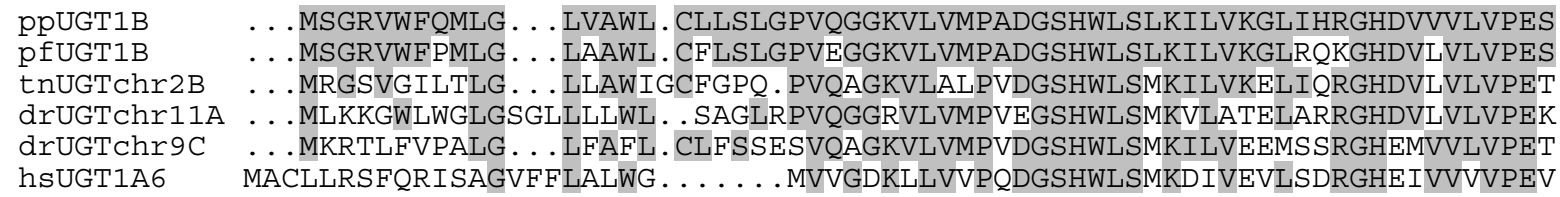

pPUGT1B SLFMHQSEDYETEVYPVSFTTEEMDATHKQLKDGLFLKQPDWTEYYVNIMRFVNFTSIHLRGCENLLENQ PfUGT1B tnUGTChr2B drUGTChr11A drUGTChr9C HLFMGESEDFKTEVYPVSYTMDEMDATYRQLKDGLFLKPPDWTEYFVNIMRFVNFTS IHLRGCESLLQNQ hSUGT1A6 SLLIKSSENYRTEIYQVPYSKEDLDGSFQALKDGLFLKPPSMADLFVNVGRLVNFTTMQVTGCESLLRNQ NILIQSSELFRTETFPVKISKEQLSKSLKGFQQGVFTRSPALMDVFVQLERLLNFTGSQVEGCESLLYNE SILIGKSGNFTTKSFRVPYSFDELNAHVDHIRKTAIEKAPRF IDIVGALGNLIOFTNMQVKACEGLLYDE NLLLKEYKYYTRKIYPVPYDQEELKNRYQSFGNNHFAERSFLTAPQTEYRNNMIVIGLYFINCQSLLQDR

pPUGT1B pfUGT1B tnUGTChr2B drUGTChr11A drUGTChr9C hsUGT1A6

PLMSRMRGMGFDIVLTDPFFPCGALVGNIFSI PVVNFLRGLPCGLDMKVNKCPSPPSYI PVPYSGNTNIM PLMSRMKGRGFDIVLTDPFFPCGALVGHIFS I PVVNFLRSLPCGLDLKVNKSPSPLSYVPVTYSGHTNIM PLMTRLREQGFEAVLTDPFLPCGS IVSHLFNI PAVYFLRGLPCELDSKANQCPAPPSYVPTSFSGNSDVM PLMRKLKEQNFELMLTDPFLPCGPI IATALGVPAVYFLRGMPCGIDLLASQCPSPPSYVPRFHSGTSDKM PLMKSLRDMKFDALLTDPFLPCGSVIADYFS I PAVYFLRGI PCRLDEAAAQCP SP PSFI PRFFTGYTDKM DTLNFFKESKFDALFTDPALPCGVILAEYLGLPSVYLFRGFPCSLEHTFSRSPDPVSYIPRCYTKFSDHM

ppUGT1B pfUGT1B TFPORVINMAMTVVESYOCSLLYGHYDEMVSKYVGNNMDYRTLLSHGALWLIRNEFTLDWPRPLLPNMVL TFPQRVTNMALSVVESYSCSLLFGHFDELVSKHLGNDMDYGTLLSHGALWLIRNDFTLDWPTPLLPNMVL tnUGTChr2B TFPQRVKNMLMYLVQSYLCNVMYHEFDRLVTRHMSDIQSYRELISRGAFWLLRYDFTFEHPKPVMPNTAF drUGTChr11A NFVERIRNFFMSGFELVLCKVMYASFDELAARYLKKDVTYKEI I GRGALWLHRYDFTFEYPRPIMPNMVF drUGTChr9C hSUGT1A6 TFPQRMINTFMTVFEKYLCHQLFASFDELATRYLKKDTSYAELLGHGAVWLLRYDFSFEYPKPQMPNMVQ TFSQRVANFLVNLLEPYLFYCLFSKYEKLASAVLKRDVDI ITLS . EVSVWLLRYDFVLEYPRPVMPNMVF

pPUGT1B pfUGT1B tnUGTchr2B drUGTChr11A IGGINCAEKKKNASLPADLEEFVQGSGDDGF I IFTLGSMLPDMPQEKAQHFLDAFRQI PQRVVWRYAGDP IGGINCAEKKKKDPLPADLEEFVQGSGDHGF IVFTLGSMLPDMPEEMAQHFLDAFRKI PQRVVWRFAGAP IGGINCA . KK. APLPADLEEFVNGSGDDGF IVFTLGSMVDNMPEEKAKQFFDAFAQI PQRVLWRYNGAV IGGINCQ . . . KSAEISAEVEEFVNGSGEHGIVVFSLGSLVSSMPKEKADIFFKAFSMI PQRVLWRYTGEI drUGTChr9C hsUGT1A6 IGGINCA . . . KRAPLTKELEEFVNGSGEHGFVVFTLGSMVSQLPEAKAREFFEAFRQI PQRVLWRYTGPV IGGINCK . . KRKDLSQEFEAYINASGEHGIVVFSLGSMVSEIPEKKAMAIADALGKNPQTVLWRYTGTR

PDUGT1B pfUGT1B tnUGTchr2B drUGTChr11A drUGTChr9C hSUGT1A6

PKGLPKNVRLMKWLPQKELLAHPKARLFLTHGGSHSVYEGICNAVPMLMFPLFAEQGDNGLRMVTRGAAE PKGLPKNVKLMKWLPQRDLLAHPKAKLFLTHGGSHSVFEGICNAMPMLMFPLFAEQGDNGLRMATRGVAE PENAPKNVKVMKWLPQNDLLAHPKAKVF ITHGGIHGI YEG I CNGVPMLMF PLFGDQ IDNVPRMI IRGVAE PNNVPENVKLMKWLPQNDLLGHPKARAFITHGGTHGIYEGI CHGVPMVMLPLFGDQADNVHRVATRGVGV PENAPKNVKLMKWLPQNDLLGHPKVRAFVTHGGSHGIYEGICNGVPMVMLPLFGDQGDNAQRLVSRGVAE PSNLANNTILVKWLPQNDLLGHPMTRAF I THAGSHGVYES I CNGVPMVMMPLFGDQMDNAKRMETKGAGV

ppUGT1B pfUGT1B tnUGTChr2B drUGTChr11A drUGTChr9C hsUGT1A6

TLNIYDVTSDNLLAALNKILKNKSYKEKITEMSQIHHDRPVAPLDLAIFWTEVVIRHKGASHLRVAAHEL TIFIYEVTSDTLLAA INKILQNKSYKEKMVELSEI HHDRPVGPLDLAI FWTEFVIRHKGAAHLKIAAHEL TLS IYDVTSQKLVARLRKWSRTRGYKENMVTLSQLNQDRPVAPLDLAVFWTEFVMRHQGAQHLRVAAHDL ILS IHDITVETLLDALNSVINNSSYKQKMQKLSA I HNDRP IQPLDLAVFWTEFVMRHKGADHLRPAAHEL SLTIYDVTSEKLLVALKKVINDKSYKEKMMKLSAI HRDRP IEPLDLAVFWTEFVMRHKGAEHLRPAAHDI TLNVLEMTSEDLENALKAVINDKSYKENIMRLSSLHKDRPVEPLDLAVFWVEFVMRHKGAPHLRPAAHDL

pPUGT1B

pfUGT1B

tnUGTChr2B

drUGTChr11A

drUGTChr9C

hsUGT1A6

NWIQYHSLDVFGF ILLILLTVLWVTLKCCLFHARRCCRRGTAKTKSE . . . . NWVQHHSLDVIGF ILLILLTVLWVTLKCCLLSTRRCCRRGTAKTKSE . . . . . NWFQYHSLDIFCFLAVVLLTVLWVLLKCCSCCARRCLRIG . . KMKKE . . . . .

NWLQYHSLDVIGFMLLIVLIVTLAMLKCCSLCWRRCCRKTQ . KRKED . . . . . NWIQYHSLDVIGFLLLILLTVIFVTVKSCMFCFRKCFKTSQ. KKKKA . . . . . TWYQYHSLDVIGFLLAVVLTVAFITFKCCPYGYPKCLGKKGRVKKAHKSKTH

\section{Figure 1. Comparison of fish and human UGT1 family proteins}

Shaded residues indicate identity to plaice UGT1B. Pp, plaice; Fp, flounder; tn, Tetraodon; dr, zebrafish; hs, human. Chromosome number is indicated where applicable.

chromosome 15, contained a single related gene that encoded a UGT in which the aglyconebinding region was encoded by five exons (ie "exon 1" was divided) and the glycone-binding region was encoded by 4 exons of equivalence to those in the other genes. It should be noted that at this time it is not possible to predict the exact numbers and structures of T. nigroviridis UGT genes because the genome sequence is incomplete and the EST resource for this species is relatively small. In the zebrafish genome, two loci bearing homologous genes were detected on chromosomes 9 and 11. Their genic organization differed from that in pufferfish since the genes were organised like the mammalian UGT1A locus, with multiple exon 1 sequences repeated upstream of a single set of exons 2 to 5 . This suggests that the zebrafish 
would express a variety of transcripts, similar to mammalian UGT1A transcripts. Indeed, this picture is confirmed for the gene on zebrafish chromosome 9 (Fig 2) where three distinct full length transcripts derived from this locus by splicing of the discrete exon 1's to the common 4 exon UDP-glucuronic acid-binding domain are represented in the EMBL/Genbank EST databases. We therefore conclude that this gene on chromosome 9 encodes several functional UGTs organised and expressed like the mammalian UGT1A locus. The gene on zebrafish chromosome 11, although having a similar organisation to that on chromosome 9, thus far is only represented by a single full length cDNA (EST) which corresponds to an exon encoding an aglycone-binding domain proximal to the four exons encoding the UDPglucuronic acid-binding domain (Fig 2).
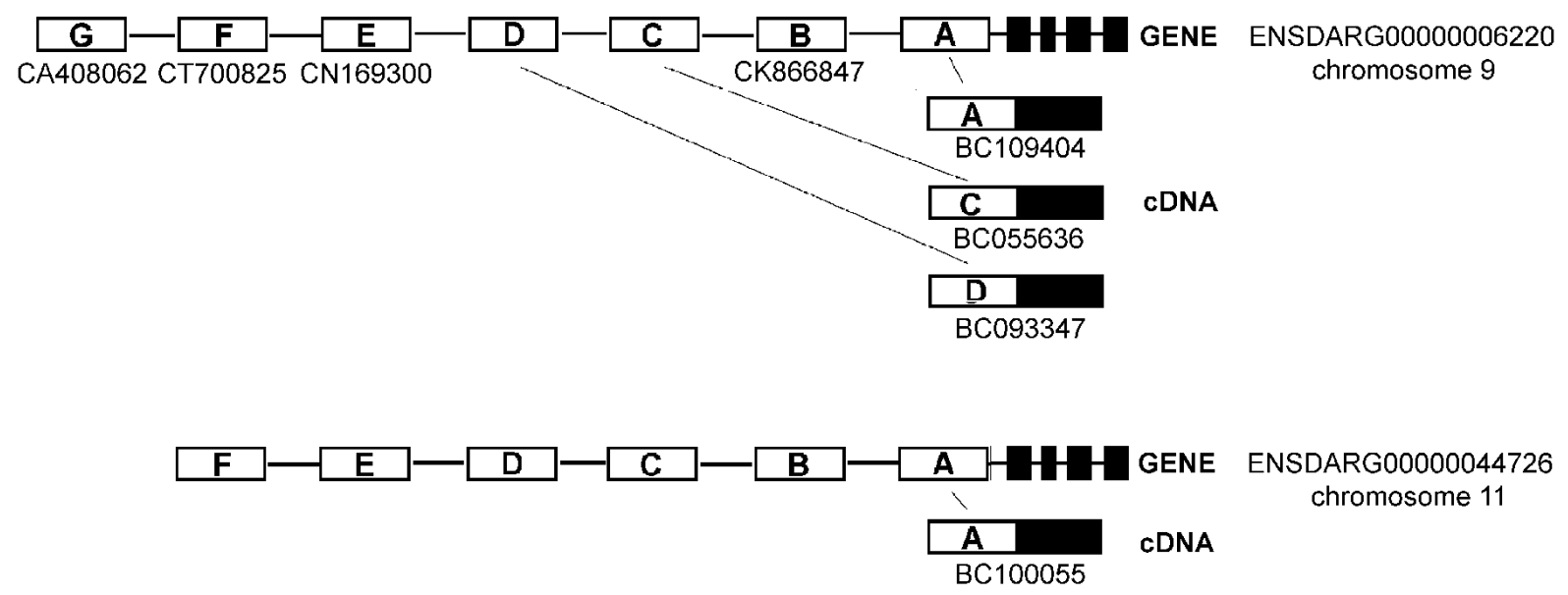

Figure 2. Organisation and expressed transcripts of the zebrafish UGT1B loci.

Ensembl gene number and chromosome is indicated. Filled boxes represent the common exons 2-5 and open boxes represent the alternative aglycone encoding exons (A to $G$ ) identified in the zebrafish genome by TBLASTX of plaice UGT1B (see text). Full-length cDNAs deposited in the EMBL/Genbank databases are indicated with accession numbers. ESTs encoding other alternative exons are also indicated by EMBL/Genbank accession number. All ESTs and cDNAs share over 99\% identity over 400 nucleotides or more with the corresponding gene sequences.

Phylogenetic trees derived from multiple comparisons of aglycone coding sequence from all of these genes (Fig 3) showed that flatfish UGT clustered with pufferfish genes on chromosome 2. The pufferfish gene on chromosome 15 clustered most closely with a subset of zebrafish aglycone coding sequences on chromosome 11. The mammalian UGT1A genes form a separate branch from all fish genes. The bootstrap analysis of the neighbour joining tree confirm the reliability of these relationships, resulting in robust tree topologies with regard to the placement of plaice and mammalian UGT sequences.

In view of the possibilities for alternative gene structures indicated by zebrafish and pufferfish genome analysis and EST evidence, a Southern blot of plaice DNA was carried out. Plaice genomic DNA was digested with various restriction enzymes, alone or in combination, and probed with exon1 (the sequence encoding the aglycone-binding domain) of the plaice cDNA. The results show a strong hybridisation to one or two genomic fragments in each digest. The sizes of these fragments are consistent with the known gene structure and sequence for plaice UGT1B (George et al., 1998). This is particularly obvious after digestion with DraI which resulted in a single hybridising fragment corresponding to the size predicted from the positions of restriction sites flanking exon 1 observed in the genomic sequence (Fig. 4). 


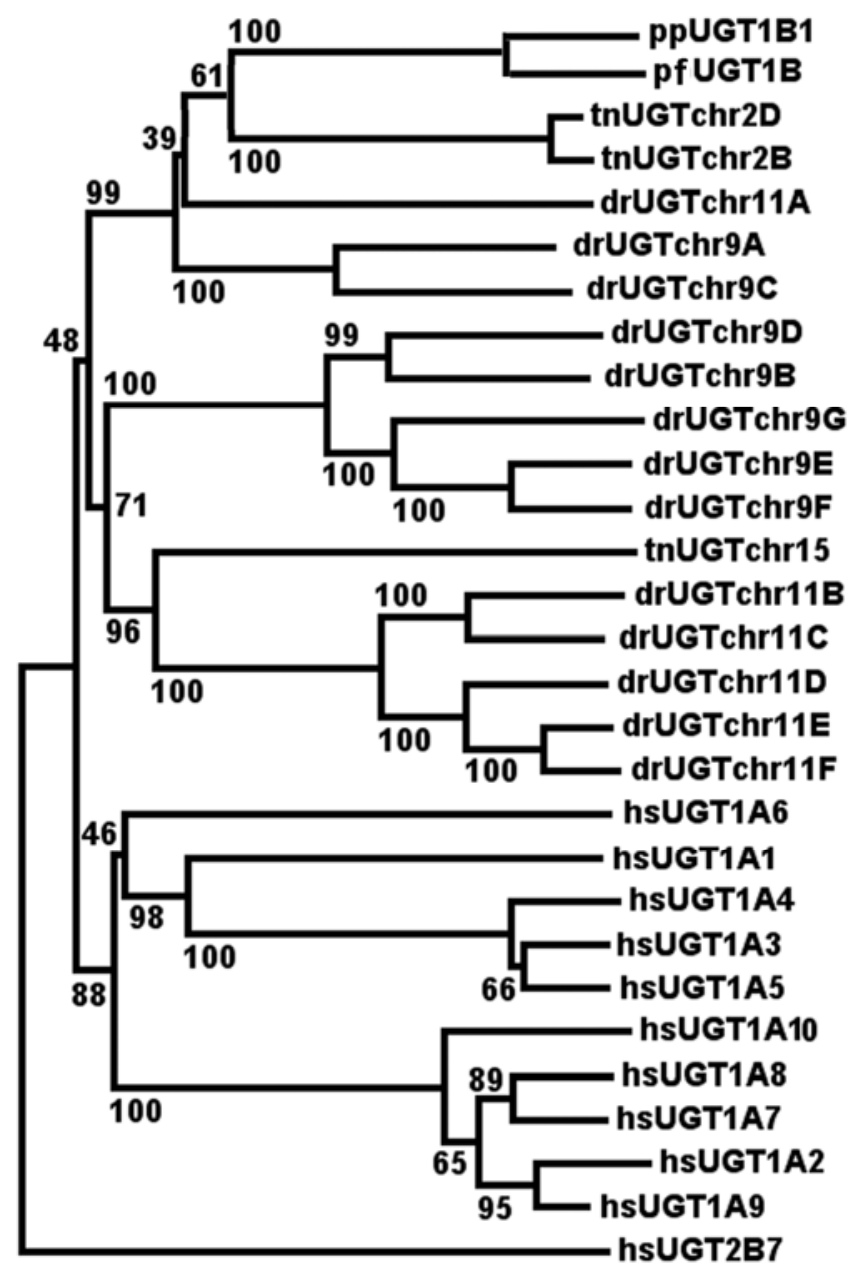

Figure 3. Phylogenetic comparison of Fish and Mammalian UGT1 proteins

Tree was constructed from a Clustal multiple alignment of the deduced polypeptide sequences of UGT aglycone binding coding sequences. Coding sequences were taken from Ensembl genomic databases. Numbers represent the percentage of times the tree topology was returned after computing trees from 1000 random samplings of the alignment. Hs, human; dr, zebrafish, tn, Tetraodon nigrividridis; pp, plaice; pf, flounder.

However in some digests there was weaker hybridisation to fragments which cannot be explained by reference to the UGT1B gene. These might suggest the presence of other more distantly related UGTs in the plaice genome.

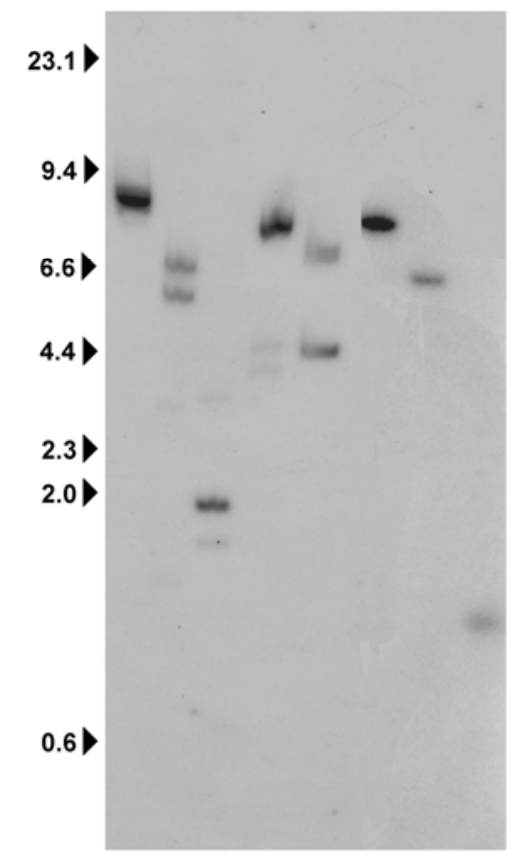

Figure 4. Southern blot of plaice genomic DNA.

Plaice DNA was restriction digested, resolved by agarose gel electrophoresis and blotted to membrane. Membrane was hybridised to the aglycone-binding coding domain of plaice UGT1B cDNA. Lanes 1, plaice DNA digested with BamHI; 2, HindIII; 3, PstI; 4, EcoRI; 5, EcoRV; 6, EcoRI pluse SalI; 7, EcoRI plus SstI; 8, DraI. Migration of standards (X10 3 base pairs) are indicated.

Tissue expression of plaice and flounder UGT and effect of xenobiotics 
Plaice and flounder showed similar UGT tissue expression profiles (Fig 5). Highest expression was observed in the liver followed by intestine of both species and lowest in red and white muscle, heart and brain. Kidney, gill, and adipose expressed intermediate levels of UGT mRNA in both species. Flounder injected intraperitoneally with prototypical chemical contaminants showed increased expression of UGT after Arochlor and lindane treatment but not after perfluoroctanoic acid or 3-methylcholanthrene. The expression of CYP1A was also measured as a comparison and it was induced up to 80-fold in Arochlor, compared to 4-fold for UGT. CYP1A was also increased after lindane and 3MC treatment, although.only up to six fold (Fig 6A). Isolated flounder hepatocytes treated with benzo(a)pyrene showed 11.6fold increases in UGT1B mRNA compared to controls, and $>50$-fold increases in CYP1A mRNA. There was no effect of ethynylestradiol treatment on either UGT1B or CYP1A mRNA in flounder hepatocytes (Fig 6B).

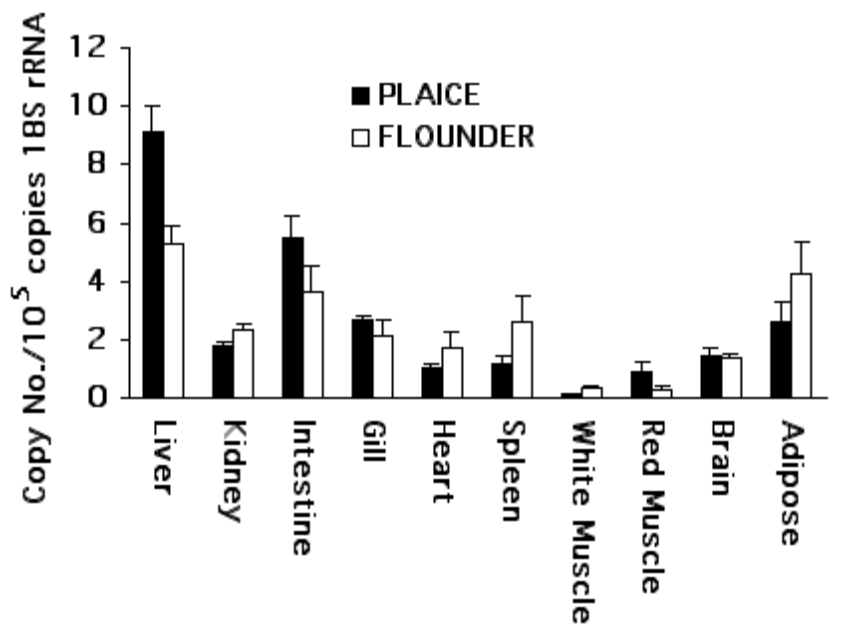

Figure 5. Tissue expression of UGT1B

Generated from quantitative RT-PCR as described in the text. UGT mRNA copy number is normalized to copy number of 18S RNA Error bars represent standard deviations of the mean of triplicate determinations from a single individual plaice or flounder.
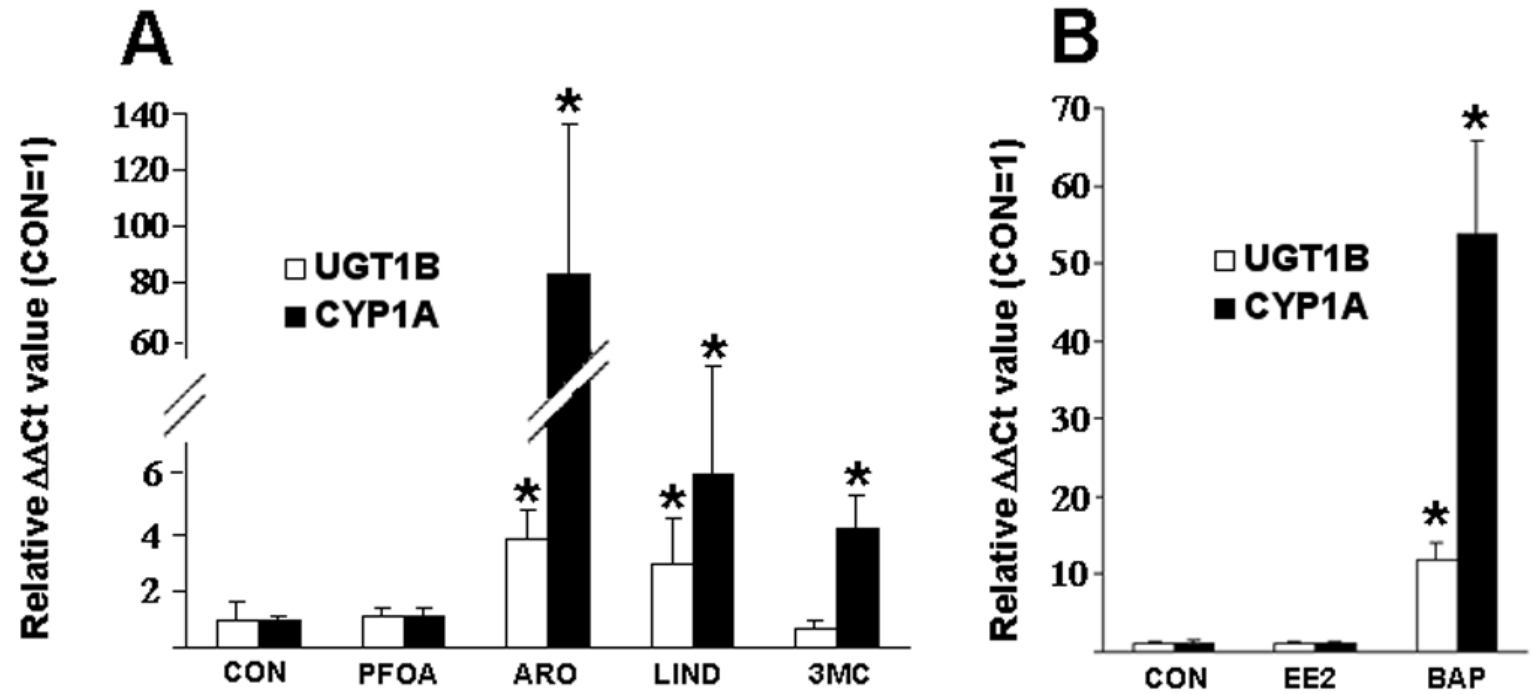

Figure 6. Expression of flounder UGT1B mRNA after treatment with prototypical chemical pollutants.

Figure is generated from quantitative RT-PCR data as described in the text. Expression levels are relative to control, set to 1, in each case and are calculated according to the "delta-delta Ct" method as described in the text. Panel A: Effects of IP injection of perflurooctanoic acid (PFOA, n=5 individuals), Arochlor 1254 (ARO, n=5 individuals), lindane (LIND, $n=5$ individuals) or 3-methycholanthrene (3MC, $n=4$ individuals). Vehicle control is olive oil (CON, n=3 individuals). Panel B: Effects of ethynylestradiol (EE2) or benzo(a)pyrene (BAP) on UGT expression in isolated flounder hepatocytes. Vehicle control is DMSO. Results derived from triplicate treatments of hepatocytes (livers) pooled from 6 individuals. Asterisks denote significant difference from control $(\mathrm{P}<0.05$, Students T-test).

\section{Embryolarval expression of plaice UGT1B}


Levels of UGT1B mRNA were undetectable in eggs and fertilised eggs and there was a massive surge in expression very early in embryonic development, between gastrulation and early somitogenesis (Fig. 7). Precisely the same timing was observed in all batches of embryos analysed. Once myotomes were synthesized (observed visually and also by troponin I mRNA expression) there was a steady decline in level throughout embryonic development, hatching until yolk sac resorption, after which there was a transient much less marked elevation during the early days of feeding.

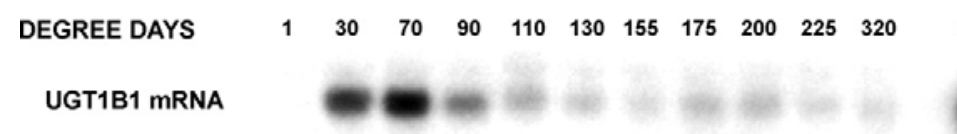

TOTAL RNA

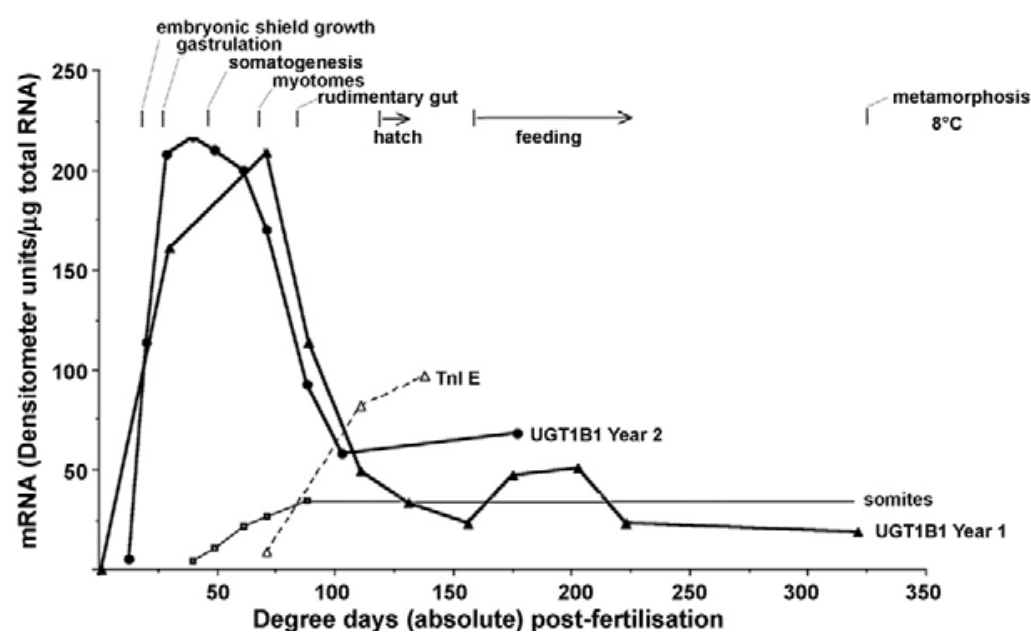

Figure 7. Embryolarval expression of Plaice UGT1B.

$10 \mu \mathrm{g}$ samples of total RNA from whole embryos/ larvae or $1 \mu \mathrm{g}$ liver RNA were electrophoresed an hybridised to the coding region of exon1 of plaice UGT1B as a probe . Upper panels: autoradiogram of northern blot and agarose gel visualised by UV transilluminattion. Lower panel: Quantified UGT1B mRNA and embryolarval staging. Myotome formation visualised by expression of muscle Troponin I mRNA.

\section{Catalytic Activity of plaice UGT}

A cDNA containing a full length coding sequences were obtained by RT-PCR of total liver RNA from a single male plaice using primers directed to sequences immediately upstream and downstream of the initiation and termination codons of the previously isolated plaice UGT1B gene. A variety of substrates were tested for their ability to undergo conjugation with UDPGA by transiently expressed plaice UGT1B (Fig 8). Most activity was observed with 1naphthol as acceptor substrate and there was also some significant but lower level activity towards estradiol and testosterone. No activity could be detected with bilirubin, or any of the bile acids tested.

\section{DISCUSSION}

We have previously reported that the exon structure of the plaice UGT1 gene is similar to mammalian UGT1A family genes in that it comprises five exons with junctions in identical positions. However, the mammalian UGT1A gene complex is composed of a multiplicity of closely related exon1 regions which are situated upstream of exons 2 to 5 . The various 


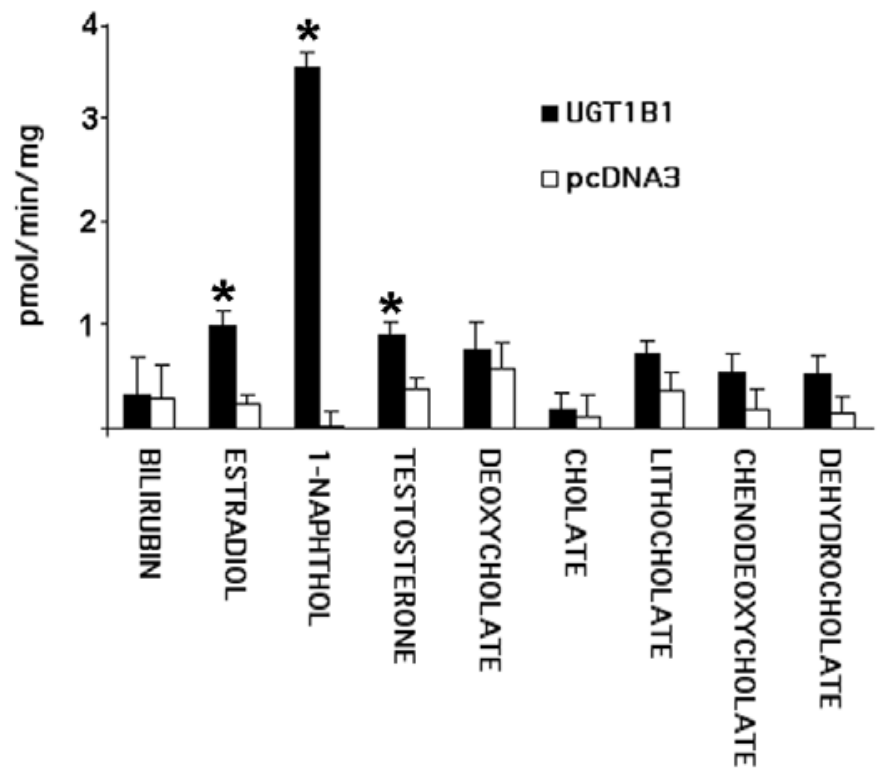

Figure 8. Plaice UGT1B is a phenol conjugating UDP-glucuronosyltransferase Activity of COS7 cells transfected with plaice UGT1B cDNA or pcDNA3 (control without UGT1B) and assayed with both acceptor substrate and UDP-glucuronic acid (filled bars). All assays were performed in triplicate. Asterisks indicate significant difference $(\mathrm{P}<0.05$, Students $\mathrm{T}$-test $)$ of tests from both controls.

mammalian UGT1A proteins are then translated from transcripts, comprising common exons 2 to 5 and a variable exon1, arising from alternative promoter activation and alternative splicing (Owens and Ritter, 1992; Owens et al, 2005). Searches of the sequenced genomes of zebrafish and pufferfish revealed the presence of closely related genes to plaice UGT1. In the zebrafish, at two loci, several exon 1 sequences are repeated upstream of a single set of exons 2 to 5 . Based on EST and cDNA evidence these genes give rise to a variety of alternative transcripts, similar to mammalian UGT1A transcripts. Interestingly, or perhaps coincidentally, recent estimates of the number of aglycone coding sequences, including pseudogenes, at the human UGT1A locus, thirteen in total (Gong et al., 2001) matches the total number of aglycone-binding domain coding sequences found at both zebrafish loci. In pufferfish the situation is less complex, having up to four entire, closely related and duplicated UGT genes at one locus and a single related UGT gene of different structure at another genomic location. It seems likely that in plaice the situation is less complex still. Given the similarity of the duplicated pufferfish genes and indeed the various zebrafish genes, it might have been expected that Southern blotting of the plaice genome with a probe for the aglycone-binding coding sequence would have revealed multiple genes. In fact Southern blotting evidence suggests that the plaice UGT gene isolated here may not duplicated, although the existence of other less closely related UGT1 genes cannot be ruled out, given the presence of additional weakly hybridising fragments. Screening of a flounder genomic library also only resulted in the identification of a single gene for UGT1 which is $91.8 \%$ identical to the plaice gene across the entire (exon and intron) sequence indicating that this flounder gene is the homologue of the original plaice gene. On the other hand it is interesting that there is a second more distantly related UGT1 locus in pufferfish and since plaice belong to the same clade as the pufferfish (Acanthopterygii) and it is therefore likely that plaice will posses a homologue of this gene. In this regard it is worth noting that it is possible to separate phenolfrom bilirubin-conjugating activity in solubilised plaice microsomes, which suggests that plaice possess an as yet unidentified bilirubin conjugating UGT isoform (Clark et al., 1992).

Taken together the genomic data suggests that the organisation of fish UGT1-related genes will depend on the order to which a fish species belongs. Thus Cypriniform species, as exemplified by the zebrafish, will contain UGT genes organised in a similar alternative promoter use/ alternative spliced manner to those of mammals, whilst Acanthopterygians 
(containing pufferfish and flatfish) will have complete, and possibly duplicated UGT genes. It is also notable that although the zebrafish genes share a similar organisation to mammalian UGT1A genes they are more closely related to the genes found in Tetraodon or plaice. This suggests that mammals and zebrafish have independently evolved a multiplicity of UGT1 exon1s from a common ancestral gene, whilst pufferfish have duplicated entire UGT1B genes.

The evidence for the identity of this plaice gene as coding for a functional UGT is first inferred from the structural similarities of the predicted flatfish polypeptides with mammalian UGT proteins. Direct evidence for the plaice gene encoding a functional UGT comes from heterologous expression. The activity of the plaice UGT1 protein was determined for a variety of substrates after transient expression of the cDNA into cos7 cells. Although it is possible from the results that the plaice UGT enzyme has some activity towards estradiol and testosterone, this is low in comparison to activity towards 1-naphthol. It should be noted that plaice liver microsomes exhibit very low steroid and bilirubin-conjugating activities when compared to mammalian species and these activities are in the order of 100 -fold lower than the activity for 1-naphthol conjugation (Clark et al., 1991). The relatively higher activity toward 1-naphthol in cos7 cells transfected with the plaice cDNA demonstrates that the expressed product is a UGT and has greater activity toward phenols than bilirubin, steroids or bile acids. In addition, given the 1-naphthol-conjugating activity, the low, or lack of activity toward steroids and bilirubin, and the tissue expression profile of the plaice UGT described here, we suggest that we have isolated the gene corresponding to a purified phenolconjugating plaice UGT protein described previously (Clark et al., 1992a). This protein, like the expressed UGT1B cDNA, possessed negligible activity toward bilirubin and only very low activity toward steroid substrates, but was an efficient catalyst of 1-naphthol conjugation. Moreover UGT1B mRNA has a wide tissue expression profile which is in concordance with the tissue profile of 1-naphthol conjugation in this species (Clarke et al., 1991; 1992a).

The flounder UGT gene is responsive to prototypical environmental contaminants. The induction of UGT and CYP1A mRNA in flounder IP injected with Arochlor and in hepatocytes treated with benzo(a)pyrene suggests that it is regulated similarly to the CYP1A gene, presumably via the Ah receptor. However it is also apparent that, although benzo(a)pyrene is an effective inducer in isolated hepatocytes, 3-methylcholanthrene, another polyaromatic hydrocarbon, is ineffective in IP injected fish. It has also been shown that Arochlor 1254, but not 3-methycholanthrene was an effective inducer of the previously characterized plaice phenol-conjugating UGT protein (Clarke et al., 1992b; George, 1994). It is noticeable that in IP-injected flounder the magnitude of CYP1A response after 3MC treatment is considerably less than that for Arochlor 1254, again suggesting that 3methylcholanthrene is not a strong Ah receptor agonist in flounder. Nevertheless the response of flounder UGT to Arochlor and benzo(a)pyrene and the lack of effect of other prototypical contaminants such as ethynylestradiol or perfluorooctanioc acid suggests that environmental flounder UGT mRNA induction may be a useful biomarker of exposure for Ah receptor interacting pollutants such as polyaromatic hydrocarbons and planar polychlorinated hydrocarbons.

The finding that there was a massive expression of UGT1B very early in development was suprising and contrasts that observed for the mRNAs of other xenobiotic metabolising and conjugative enzymes in plaice where levels rise after the onset of myogenesis (Hodgson and George 1988 and unpublished results). To our knowledge, expression at very early stages of mammalian embryonic development has not been documented . Glucuronidation of 1naphthol (by UGT1A6) can be detected in human foetal liver by mid trimester (Leakey 
1987, Coughtrie et al., 1988) whilst conjugation of further substrates (including bilirubin by UGT1A2/1A4) is delayed until after birth (Coughtrie et al., 1988). In rats expression of UGTs is grouped in three "clusters" - late foetal (conjugation of planar phenols), neonatal (conjugation of bilirubin and testosterone) and post-weaning (conjugaton of androsterone) (Matsui and Watanabe, 1982; reviewed by Burchell and Coughtrie, 1989). This very high expression of UGT1B, the plaice UGT1A6 homologue, at such an early developmental stage clearly warrants further investigation since an essential role is implied and the natural substrate is unknown, moreover, since we have detected the occurrence of a null allele in this species (George and Leaver , 2002) this could have important implications for survival.

In summary genes related to this plaice UGT1B, which appears to encode a planar phenolconjugating isoform, are highly represented in fish genomes but their genic organization differs between fish orders. During searches of fish genomes we have also found a number of UGT2 family-related genes (George and Taylor, 2002) and another, highly numerous group of UGT-related sequences which are clearly not represented in mammals (in prep. not shown). Thus, taking all the evidence together, we suggest that UGT1B should be the designation for all of the piscine UGT genes described here and they should be considered as fish homologues of mammalian UGT1A genes.

\section{Acknowledgement and Dedication}

This work was financially supported by grant GR3/10860 from the Natural Environment Research Council, UK. EB was supported by a Cooperative Research Program Fellowship awarded by the OECD. Dedicated to the memory of DG.

\section{REFERENCES}

Burchell, B., Coughtrie, M.W.H. (1989) UDP-Glucuronosyltransferases. Pharmac. Ther. 43, 261-289.

Burchell, B., McGurk, K., Brierley, C. H., Clarke, D. J. (1997) in Biotransformation (Guengerich, F. G., ed.), pp. 401-435, Comprehensive Toxicology, vol. 3 (Sipes, I. G., McQueen, C. A. and Gandolfi, A. J., series eds.), Elsevier Science, Oxford

Chen, S.J., Beaton, D., Nguyen, N., Senekeo-Effenberger, K., Brace-Sinnokrak, E., Argikar, U., Remmel, R.P., Trottier, J., Barbier, O., Ritter, J. K., Tukey, R. H. (2005) Tissue-specific, inducible, and hormonal control of the human UDP-glucuronosyltransferase-1 (UGT1) locus. J. Biol. Chem. 280, 37547-37557.

Clarke, D.J., Burchell, B, George, S. G. (1992) Diffrential expression and induction of UDPglucuronosyltransferase isoforms in hepatic and extrahepatic tissues of a fish, Pleuronectes platessa: Immunochemical and functional chracterisation. Toicol. Appl. Pharmacol. 115, 130136.

Clarke, D.J., Burchell, B. (1994) The uridine diphosphate glucuronosyltransferase multigene family: function and regulation. Handbook of Experimental Pharmacology, Vol. 112, chapter 1. Ed. Kauffman, F.C. Springer-Verlag, Berlin.

Clarke, D. J., George, S. G., Burchell, B. (1991) Glucuronidation in fish. Aquat. Toxicol. 20, 35-56 
Clarke, D. J., George, S. G., Burchell, B. (1992) Multiplicity of UDPglucuronosyltransferases in fish. Biochem. J. 284, 417-423

Coughtrie, M.W.H., Burchell, B., Leakey, J.E.A., Hume, R. (1988) The inadequacy of perinatal glucuronidation. Molec. Pharmac. 35, 585-591.

Dutton, G. J., (1980) Glucuronidation of Drugs and Other Compounds, CRC Press, Boca Raton, FL.

Emi, Y., Ikushiro, S., Iyanagi, T. (1995) Drug-responsive and tissue-specific alternative expression of multiple first exons in rat UDP_glucuronosyltransferase family 1 (UGT1) gene complex. J. Biochem. 117, 392-399.

George, S. G. (1994) In Aquatic Toxicology:Molecular, Biochemical and Cellular Perspectives (Malins, D. C., and Ostrander, G. K., eds), Lewis Publishers, Boca Raton, Ann Arbor, London, Tokyo.

George, S. G., Leaver, M.J., Wright, J. (1998) Structural studies of a UDPglucuronosyltransferase gene from the plaice (Pleuronectes platessa) Mar. Env. Res. 46, 3337

George, S.G., Leaver, M. (2002) Allelic variations in the plaice UGT1B1 gene. Mar. Env. Res. 54, 259-262.

George, S.G, Taylor, B. (2002) Molecular evidence for multiple UDPglucuronosyltransferase gene families in fish. Mar. Env. Res. 54, 253-257.

Gong, Q.H., Cho, J.W., Huang, T., Potter, C., Gholami, N., Basu, N.K., Kubota, S., Carvalho, S., Pennington, M.W., Owens, I.S., Popescu, N.C. (2001) Thirteen UDP glucuronosyltransferase genes are encoded at the human UGT1 gene complex locus. Pharmacogenetics 11, 357-368.

Green, M. D., Oturu, E. M., Tephly, T. R. (1993) Substrate-specificity of a stably expressed human hepatic UDP- glucuronosyltransferase (UGT 2B8). Faseb Journal 7, A52

Green, M. D., Oturu, E. M., Tephly, T. R. (1994) Stable expression of a human liver UDPglucuronosyltransferase (UGT2B15) with activity toward steroid and xenobiotic substrates. Drug Metabolism And Disposition

22, 799-805

Green, M. D., Clarke, D. J., Oturu, E. M., Styczynski, P. B., Jackson, M. R., Burchell, B., Tephly, T. R. (1995) Cloning and expression of a rat liver phenobarbital-inducible UDPglucuronosyltransferase (2B12) with specificity for monoterpenoid alcohols. Archives Of Biochemistry And Biophysics

322, 460-468

Guillemette, C. (2003) Pharmacogenomics of human UDP-glucuronosyltransferase enzymes. Pharmacogenomics J. 3, 136-158. 
James, M. O. (1987) Conjugation of organic pollutants in aquatic species. Environ. Health Perspect. 71, 97-103

Leakey, J.E.A., Hume, R., Burchell, B. (1987) Development of multiple activities of UDPglucuronosyltransferase in human liver. Biochem. J. 245, 859-861.

Leaver, M. J., Clarke, D. J., George, S. G. (1992) Molecular studies of the Phase II xenobiotic conjugative enzymes of marine Pleuronectid flatfish. Aquat. Toxicol., 22 265-278

Leaver, M. J., Wright, J., George, S. G. (1997) Structure and expression of a cluster of glutathione S-transferase genes from a marine fish, the plaice (Pleuronectes platessa). Biochem. J. 321, 405-412

Mackenzie, P.I., Bock, K.W., Burchill, B., Guillemette, C., Ikushiro, S., Iyanagi, T., Miners, J.O., Owens, I.S., Nebert, D.W. (2005) Nomenclature update for the mammalian UDP glycosyltransferase (UGT) gene superfamily. Pharmacogenetics and Genomics, 15, 677-685.

Mackenzie, P.I., Gregory, P. A., Gardner-Stephen, D.A., Lewinsky, R.H., Jorgenson, B.R., Nishiyama, T., Xie, W., Radominska-Pandya, A. (2003) Regulation of UDP glucuronosyltransferase genes. Curr. Drug Metab. 4, 249-257.

Mackenzie, P. I., Owens, I. S., Burchell, B., Bock, K. W., Bairoch, A., Belanger, A., FournelGigleux, S., Green, M., Hum, D. W., Iyanagi, T., Lancet, D., Louisot, P., Mulder, G. J. (1992) Glucuronidation and its role in regulation of biological-activity of drugs. Annual Review of Pharmacology and Toxicology

32, 25-49

Mackenzie, P.I., Owens, I..S, Burchell, B., Bock, K.W., Bairoch, A., Belanger, A., FournelGigleux, S., Green, M., Hum, D.W., Iyanagi, T., Lancet, D., Louisot, P., Magdalou, J., Chowdhury, J. R., Ritter, J. K., Schachter, H., Tephly, T. R., Tipton, K. F., Nebert, D. W. (1997) The UDP glycosyltransferase gene superfamily: recommended nomenclature update based on evolutionary divergence. Pharmacogenetics 7, 255-269

Matern, H., Heinemann, H., Matern, S. (1994) Radioassay of UDP-glucuronosyltransferase activities toward endogenous substrates using labeled UDP-glucuronic acid and an organic solvent extraction procedure. Analytical Biochemistry 219, 182-188

Matsui, M., Watanabe, H.K. (1982) Developmental alteration of hepatic UDPglucuronsyltransferase and sulfotransferase activity towards androsterone and 4-nitrophenol in Wistar rats. Biochem. J. 204, 441-447.

Meech, R. \& MacKenzie, P. I. (1997) Structure and function of uridine diphosphate glucuronosyltransferases. Clinical And Experimental Pharmacology And Physiology 24, 907-915

Morrison, H., Young, P., George, S. G. (1986) Conjugation of organic compounds in isolated hepatocytes from a marine fish, the plaice, Pleuronectes platessa. Biohem. Parmacol. 34, 3933-3938

Mulder, G. J. (1992) Glucuronidation and its role in the regulation of biological activity of drugs. Ann. Rev. Pharmacology Toxicology 32, 25-49 
Running title: Piscine UDP-glucuronosyltransferase gene

Owens, I.S., Basu, N. K., Bannerjee, R. (2005) UDP-glucuronosyltransferases: Gene structures of UGT1 and UGT2 families. Methods Enzymol. 400, 1-22.

Owens, I. S. \& Ritter, J. K. (1992) The novel bilirubin phenol UDP-glucuronosyltransferase ugt1 gene locus - implications for multiple nonhemolytic familial hyperbilirubinemia phenotypes Pharmacogenetics 2, 93-108

Pfaffl, M. W. (2001) A new mathematical model for relative quantification in real-time RTPCR. Nucleic Acids Res. 29, E45.

Pirrit, L., Healey, R., Assheton, H., Leaver, M.J., George, S. G. (1995) Cloning of the major 3-MC inducible Phase I and Phase II detoxification enzymes of plaice liver. Mar. Environ. Res. 39, 15-19

Radominska-Pandya, A., Bratton, S., Little, J. M. (2005) A historical overview of the heterologous expression of mammalian UDP-glucuronosyltransferase isoforms over the past twenty years. Curr. Drug Metab. 6, 141-160.

Radominska-Pandya, A., Oussine, M., Fournel-Gigleux, S., Magdalou, J. (2005) Structure of UDP-glucuronosyltransferases in membranes. Methods Enzymol. 400, 115-147

Satou, N., Nei, M. (1987) The neighbor-joining method: a new method for reconstructing phylogenetic trees. Molecular Biology and Evolution, 4, 406-425.

Senay, C., Ouzzine, M., Battaglia, E., Pless, D., Cano, V., Burchell, B., Radominska, A., Magdalou, J., FournelGigleux, S. (1997) Arginine 52 and histidine 54 located in a conserved amino-terminal hydrophobic region (LX2-R52-G-H54-X3-V-L) are important amino acids for the functional and structural integrity of the human liver UDP- glucuronosyltransferase UGT1*6. Molecular Pharmacology

51, 406-413

Tukey, R. H., Strassburg, C. P. (2000) Human UDP-glucuronosyltransferases: Metabolism, expression and disease. Ann. Rev. Pharmacol. Toxicol. 40, 581-616 